06,05

\title{
Фрустрация обменно-стрикционного сегнетоэлектрического упорядочения и рост электрической поляризации областей фазового расслоения в твердых растворах $R_{0.8} \mathrm{Ce}_{0.2} \mathrm{Mn}_{2} \mathrm{O}_{5}(\mathrm{Er}, \mathrm{Tb})$
}

\author{
(С) Б.Х. Ханнанов, Е.И. Головенчиц, В.А. Санина \\ Физико-технический институт им. А.Ф. Иофрфе РАН, \\ Санкт-Петербург, Россия \\ E-mail: sanina@mail.ioffe.ru
}

Поступила в Редакцию 27 ноября 2019 г.

В окончательной редакции 27 ноября 2019 г.

Принята к публикации 28 ноября 2019 г.

\begin{abstract}
Изучено влияние редкоземельных ионов $\left(R=\mathrm{Er}^{3+}, \mathrm{Tb}^{3+}, \mathrm{Ce}^{3.75+}\right)$ на диэлектрические свойства и электрическую поляризацию, индуцированную локальными полярными областями фазового расслоения в твердых растворах мультиферроиков $R_{0.8} \mathrm{Ce}_{0.2} \mathrm{Mn}_{2} \mathrm{O}_{5}(R=\mathrm{Er}, \mathrm{Tb})$. Обнаружено качественное отличие этих параметров от изученных ранее исходных кристаллов $R \mathrm{Mn}_{2} \mathrm{O}_{5}(R=\mathrm{Er}, \mathrm{Tb})$. Показано, что свойства полярных областей фазового расслоения, возникающих в подсистеме ионов $\mathrm{Mn}^{3+}$ и $\mathrm{Mn}^{4+}$ благодаря конечной вероятности туннелирования электронов между этими ионами различной валентности, существенным образом зависят от величин кристаллических полей, в которых эти области находятся. Оказалось, что совместное влияние ионов $\mathrm{Er}^{3+}, \mathrm{Tb}^{3+}$ и $\mathrm{Ce}^{3.75+}$ существенно изменяют кристаллическое поле в $R_{0.8} \mathrm{Ce}_{0.2} \mathrm{Mn}_{2} \mathrm{O}_{5}(R=\mathrm{Er}, \mathrm{Tb})$ по сравнению с $R \mathrm{Mn}_{2} \mathrm{O}_{5}(R=\mathrm{Er}, \mathrm{Tb})$.
\end{abstract}

Ключевые слова: мультиферроики, зарядовое упорядочение, полярные области фазового расслоения, электрическая поляризация.

DOI: 10.21883/FTT.2020.04.49123.642

\section{1. Введение}

В работе [1] представлены результаты исследования электрической поляризации, индуцированной локальными полярными областями фазового расслоения в $R \mathrm{Mn}_{2} \mathrm{O}_{5}(R=\mathrm{Er}, \mathrm{Tb})$ и их сравнение со свойствами в $R \mathrm{Mn}_{2} \mathrm{O}_{5} \quad(R=\mathrm{Gd}, \mathrm{Bi})$ [2-4]. Все эти кристаллы относятся к мультиферроикам II типа, в которых при температурах ниже температур Кюри $\left(T=T_{\mathrm{C}} \leq 30-35 \mathrm{~K}\right)$ имеется сегнетоэлектрическое упорядочение, индуцированное магнитным упорядочением с температурами Нееля $T_{\mathrm{N}} \leq 35-40 \mathrm{~K}[5,6]$. В работах [1-4] было показано, что в этих мультиферроиках в широком интервале температур, от самых низких до значительно превышающих $T_{\mathrm{C}}$, возникают локальные полярные области фазового расслоения. Эти области ниже некоторых температур, зависящих от направления осей кристаллов, и значительно превышающих $T_{\mathrm{C}}$ низкотемпературного сегнетоэлектрического упорядочения, формируют замороженное суперпараэлектрическое состояние, в котором отклик локальных полярных областей на приложенное электрическое поле имеет вид петель гистерезиса электрической поляризации с остаточной поляризацией.

Характерной особенностью $R \mathrm{Mn}_{2} \mathrm{O}_{5}$ является наличие равного количества ионов марганца $\mathrm{Mn}^{3+}$ (содержащих три $t_{2 g}$ и один $e_{g}$-электрон на $3 d$-оболочке) и $\mathrm{Mn}^{4+}$ (с тремя $t_{2 g}$ электронами на $3 d$-оболочке), что обеспечивает условия для появления диэлектрического зарядового упорядочения. Ионы $\mathrm{Mn}^{4+}$ имеют октаэдрическое кислородное окружение и расположены в слоях с $z=0.25 c$ и $(1-z)=0.75 c$. Ионы $\mathrm{Mn}^{3+}$ имеют нецентральное локальное окружение в виде пятиугольных пирамид и расположены в слоях с $z=0.5 c$. Ионы $\mathrm{R}^{3+}$ с окружением подобным $\mathrm{Mn}^{3+}$ расположены в слоях c $z=0$ [7]. Зарядовое упорядочение и конечная вероятность переноса $e_{g}$-электронов между парами ионов $\mathrm{Mn}^{3+}-\mathrm{Mn}^{4+}$ (двойной обмен $\left.[8,9]\right)$ являются ключевыми факторами, определяющими электрические полярные состояния $R \mathrm{Mn}_{2} \mathrm{O}_{5}$ при всех температурах. Низкотемпературное сегнетоэлектрическое состояние при $T \leq T_{\mathrm{C}}$ обусловлено зарядовым упорядочением вдоль оси $b$. Чередование пар соседних ионов $\mathrm{Mn}^{3+}$ и $\mathrm{Mn}^{4+}$ вдоль оси $b$ с сильным ферромагнитным обменом (двойным обменом) и слабым косвенным антиферромагнитным обменом приводит к обменной стрикции, нарушающей центросимметричность решетки вдоль оси $b$ и появлению низкотемпературного сегнетоэлектрического упорядочения [10]. Перенос же $e_{g}$-электронов между парами ионов $\mathrm{Mn}^{3+}-\mathrm{Mn}^{4+}$, расположенными в соседних слоях, перпендикулярных оси $c$, приводит к образованию локальных полярных областей фазового расслоения с иным распределением ионов $\mathrm{Mn}^{3+}$ и $\mathrm{Mn}^{4+}$ по сравнению с исходной матрицей кристалла [1-4,11,12].

Локальные полярные области формируются в $R \mathrm{Mn}_{2} \mathrm{O}_{5}$ за счет процессов фазового расслоения, аналогично манганитам $\mathrm{LaAMnO}_{3}(A=\mathrm{Sr}, \mathrm{Ca}, \mathrm{Ba})$ также содержащим ионы $\mathrm{Mn}^{3+}$ и $\mathrm{Mn}^{4+}[9,13]$. Изолированные области 
фазового расслоения образуются в матрице исходного кристалла в результате процессов самоорганизации, обусловленных конечной вероятностью туннелирования $e_{g}$-электронов между парами ионов $\left(\mathrm{Mn}^{3+}-\mathrm{Mn}^{4+}\right)$ в соседних плоскостях, перпендикулярных оси $c$. Они формируются за счет баланса сильных взаимодействий (двойного обмена (с характерной энергией $0.3 \mathrm{eV}$ ), взаимодействия Яна-Теллера $(0.7 \mathrm{eV})$ и Кулоновского отталкивания $(1 \mathrm{eV}))$ и существуют в широком диапазоне температур от низких до температур выше комнатной $[8,9,11,13]$. При низких температурах $T<60 \mathrm{~K}$ области фазового расслоения в $R \mathrm{Mn}_{2} \mathrm{O}_{5}$ представляют собой изолированные $1 D$-сверхрешетки из ферромагнитных слоев, содержащих ионы $\mathrm{Mn}^{3+}$ и $\mathrm{Mn}^{4+}$ в различных соотношениях. В них наблюдался набор ферромагнитных резонансов [14-16] и электрическая поляризация [1-4] в направлениях магнитного и электрического полей, соответственно. При температурах выше $180 \mathrm{~K}$ возникает взаимодействие между ранее изолированными локальными полярными областями, формирующее $2 D$-сверхструктуры, перпендикулярные оси $c$. В таких сверхструктурах чередуются слои исходной матрицы и областей фазового расслоения. Ширины слоев при комнатной температуре достигали 700-900 А $[2-4,11,12]$.

Замороженное суперпараэлектрическое состояние для локальных сегнетоэлектрических областей в диэлектрической центросимметричной матрице было рассмотрено ранее теоретически [17], но экспериментально впервые было обнаружено в $R \mathrm{Mn}_{2} \mathrm{O}_{5}(R=\mathrm{Gd}, \mathrm{Bi})$ [2-4]. При этом было показано, что локальные полярные области фазового расслоения в $R \mathrm{Mn}_{2} \mathrm{O}_{5}$ являются мультиферроидными и управляются внешними электрическим и магнитными полями. В этих работах полагалось, что слабо интенсивные нецентральные рефлексы, обнаруженные в структурном исследовании при комнатной температуре в ряде $R \mathrm{Mn}_{2} \mathrm{O}_{5}[18]$ относятся к таким областям.

В работах [1-4] было продемонстрировано, что в $R \mathrm{Mn}_{2} \mathrm{O}_{5}(R=\mathrm{Gd}, \mathrm{Bi}, \mathrm{Er}, \mathrm{Tb})$ электрическая поляризация обусловлена областями фазового расслоения, формирующимися в подсистеме ионов $\mathrm{Mn}$, но находящейся в различных кристаллических полях, обусловленных $R$-ионами. Именно величина и анизотропия кристаллических полей определяет температуры существования и величины электрической поляризации вдоль различных осей. При этом ион $\mathrm{Gd}^{3+}$ (основное состояние ${ }^{8} S_{7 / 2}$ ) имеет максимальный спин среди $R$-ионов и в основном $S$-состоянии слабо связан с решеткой, так как у него отсутствует спин-орбитальное взаимодействие. $\mathrm{B} \mathrm{GdMn}_{2} \mathrm{O}_{5}$ наблюдалась максимальная поляризация в низкотемпературном сегнетоэлектрическом упорядочении $[19,20]$. Основное состояние ионов $\mathrm{Tb}^{3+}\left({ }^{7} F_{6}, S=3\right.$, $L=3$ ) характеризуется большим магнитным моментом $\left(J=9.7 \mu_{B}\right)$, в который вносят вклад как спиновый, так и орбитальный моменты $(S=3, L=3$ соответственно) и имеется сильная спин-орбитальная связь. Ионы $\mathrm{Tb}^{3+}$ описываются в крайне сильном анизотропном изинговском приближении, жестко фиксирующем ориентацию их моментов в плоскости $a b$ [21]. Основное состояние иона $\mathrm{Er}^{3+}\left({ }^{4} I_{15 / 2}, S=3 / 2, L=6\right)$ также обладает большим магнитным моментом $\left(J=9.6 \mu_{B}\right)$, в который основной вклад вносит орбитальный момент и имеется сильное электрическое кристаллическое поле, которое жестко ориентирует моменты ионов $\mathrm{Er}$ вдоль оси $c$ сильной одно-ионной анизотропией [21]. Магнитные моменты $R$-ионов связаны обменным взаимодействием с ионами Mn. Кроме того, $R$-ионы сильно изменяют кристаллическое поле, в котором оказывается подсистема ионов $\mathrm{Mn}$. Ионы $\mathrm{Bi}^{3+}$ немагнитные, но сильно искажают ближайшее кристаллическое окружение благодаря наличию на их наружных оболочках уединенных пар $6 s^{2}$-электронов [22].

Целью настоящей работы является изучение твердых растворов $R_{0.8} \mathrm{Ce}_{0.2} \mathrm{Mn}_{2} \mathrm{O}_{5}(R=\mathrm{Er}, \mathrm{Tb})$ и влияния разбавления подсистемы $R$-ионов ионами Се как на низкотемпературное сегнетоэлектрическое упорядочение вдоль оси $b$, так и на электрическую поляризацию, индуцированную областями фазового расслоения. Отметим, что эти разбавленные кристаллы обладают той же симметрией (пространственная группа $\mathrm{Pbam}$ ), что и исходные кристаллы $R \mathrm{Mn}_{2} \mathrm{O}_{5}$. Для кристаллов, содержащих редкоземельные ионы Се, характерно появление переменной валентности $\mathrm{Ce}^{+3.75}$, т. е. эти ионы проявляют свойства как ионов $\mathrm{Ce}^{3+}$, так и $\mathrm{Ce}^{4+}$. В работе [23] при изучении керамических образцов $\mathrm{Bi}_{0.9} \mathrm{Ce}_{0.1} \mathrm{Mn}_{2} \mathrm{O}_{5}$ было обнаружено существование ионов Се с такой переменной валентностью. Ионы Се в изучаемых нами монокристаллах твердых растворов $R_{0.8} \mathrm{Ce}_{0.2} \mathrm{Mn}_{2} \mathrm{O}_{5}(R=\mathrm{Er}, \mathrm{Tb})$ замещают позиции ионов $\mathrm{Er}^{3+}$ и $\mathrm{Tb}^{3+}$. Напомним, что $R$-ионы в $R \mathrm{Mn}_{2} \mathrm{O}_{5}$ имеют пятиугольное кислородное окружение (искаженное шестиугольное). Согласно [24] ионные радиусы в шестиугольном окружении интересующих нас ионов следующие: $\mathrm{Ce}^{4+}-0.87, \mathrm{Ce}^{3+}-1.01$, $\mathrm{Er}^{3+}-0.89, \mathrm{~Tb}^{3+}-0.923 \AA$. Таким образом, с некими вероятностями оба иона $\mathrm{Er}^{3+}$ и $\mathrm{Tb}^{3+}$ могут замещаться ионами $\mathrm{Ce}^{4+}$ и $\mathrm{Ce}^{3+}$.

Ранее нами изучалось влияние ионов Се на диэлектрические и магнитные свойства $\mathrm{Eu}_{0.8} \mathrm{Ce}_{0.2} \mathrm{Mn}_{2} \mathrm{O}_{5}$ [11,14-16]. Было показано, что разбавление ионов $\mathrm{Eu}^{3+}$ преимущественно ионами $\mathrm{Ce}^{4+}$ приводило к увеличению концентрации областей фазового расслоения при всех температурах. Это вело к увеличению интенсивности ферромагнитнитных резонансов от слоев $1 D$-сверхрешеток, представляющих собой заряженные доменные стенки между полярными доменами сегнетоэлектрического упорядочения [14-16], а также изменяло свойства высокотемпературных областей фазового расслоения по сравнению с $\mathrm{EuMn}_{2} \mathrm{O}_{5}$.

Задачей настоящего исследования является изучение влияния разбавления ионами Се монокристаллов $R \mathrm{Mn}_{2} \mathrm{O}_{5} \quad(R=\mathrm{Er}, \mathrm{Tb})$ на диэлектрические свойства и электрическую поляризацию в широком интервале температур 5-350 K. 


\section{2. Объекты и методы исследования}

Монокристаллы $\mathrm{R}_{0.8} \mathrm{Ce}_{0.2} \mathrm{Mn}_{2} \mathrm{O}_{5} \quad(R=\mathrm{Er}, \mathrm{Tb})$ выращивались методом спонтанной кристаллизации из раствора-расплава $[25,26]$. Они имели форму пластинок толщиной $2-3 \mathrm{~mm}$ и площадью $3-5 \mathrm{~mm}^{2}$.

Для измерения диэлектрических свойств и поляризации изготавливались плоские конденсаторы толщиной 0.3-0.6 mm и площадью 3-4 mm². Диэлектрическая проницаемость и проводимость определялись с помощью измерителя импеданса Good Will LCR-819 в частотном интервале $0.5-50 \mathrm{kHz}$, в интервале температур 5-330 K.

Электрическая поляризация измерялась PUND (Positive Up Negative Down) методом [27-29]. Нами использовался адаптированный к измерениям поляризации локальных полярных областей с локальной проводимостью PUND-метод, описанный в [2-4]. PUND-метод позволяет корректно учесть вклад проводимости в измеряемую петлю электрической поляризации. Это особенно актуально при изучении разбавленных $R_{0.8} \mathrm{Ce}_{0.2} \mathrm{Mn}_{2} \mathrm{O}_{5}$ $(R=\mathrm{Er}, \mathrm{Tb})$ с повышенным фоном проводимости. $\mathrm{B}$ этом методе отклик внутренней поляризации $P$ может быть выделен при приложении серии положительных $P 1-P 2$ и отрицательных $N 1-N 2$ импульсов напряжения К измеряемому образцу. При этом регистрируются независимые кривые $(P 1-P 2)$ и $(N 1-N 2)$ отклика эффективного изменения поляризации на эту серию импульсов. PUND-метод основан на различии динамики откликов внутренней поляризации и проводимости на импульсы приложенного электрического поля $E$. При выключении импульса поляризация релаксирует значительно медленнее, чем проводимость. Временные интервалы между импульсами $P 1-P 2$ и $N 1-N 2$ должны быть такими, чтобы за это время внутренняя поляризация слабо изменилась, в то время как релаксация проводимости завершилась полностью. Истинная внутренняя поляризация вычисляется вычитанием парных импульсов $P 1-P 2$ и N41-N2 друг из друга.

\section{3. Экспериментальные данные и их анализ}

\section{1. Диэлектрические свойства и электрическая поляризация в $\mathrm{Er}_{0.8} \mathrm{Ce}_{0.2} \mathrm{Mn}_{2} \mathrm{O}_{5}$}

На рис. $1, a-f$ для $\mathrm{Er}_{0.8} \mathrm{Ce}_{0.2} \mathrm{Mn}_{2} \mathrm{O}_{5}$ (ЕСМО) приведены температурные зависимости диэлектрической проницаемости $\mathcal{E}^{\prime}$ для ряда частот вдоль осей $a, b, c$ (рис. 1, $a, c, e$ соответственно) и проводимости $\sigma$ вдоль этих же осей (рис. $1, b, d$ и $f$ соответственно). На вставке рис. 1, $c$ приведены величины $\varepsilon^{\prime}$ в большем масштабе для ряда частот в $\mathrm{ErMn}_{2} \mathrm{O}_{5}$ (EMO) и ЕСМО. Как видно, разбавление ионов $\mathrm{Er}$ ионами Се приводит к сильному нарушению низкотемпературного сегнетоэлектрического упорядочения вдоль оси $b$ и значительному росту фонового низкотемпературного значения $\varepsilon^{\prime}$. Для
ECMO бездисперсионные низкотемпературные значения $\varepsilon^{\prime} \sim 100-130$ в зависимости от ориентации осей кристалла в 5-6 раз превышают подобные значения в EMO [1]. Значительное нарастание проницаемостей $\varepsilon^{\prime}$ на различных частотах вдоль всех осей начинаются с $T \sim 175 \mathrm{~K}$. Вблизи комнатной температуры максимальное значение $\varepsilon^{\prime}$ наблюдается вдоль оси $b\left(3.5 \cdot 10^{4}\right)$. Вдоль осей $a$ и $c$ значения $\varepsilon^{\prime} \approx 1.75 \cdot 10^{4}$.

Проводимости и их анизотропия в ЕСМО отличаются от таких величин в EMO [1]. Также как в EMO мы имеем дело с действительной частью проводимости $\sigma_{1}=\omega \varepsilon^{\prime \prime} \varepsilon_{0}[30]$, которая вычисляется из диэлектрических потерь $\varepsilon^{\prime \prime}$ (измеряется тангенс диэлектрических потерь $\left.\operatorname{tg} \delta=\varepsilon^{\prime \prime} / \varepsilon^{\prime}\right)$. Здесь $\omega-$ угловая частота, а $\varepsilon_{0}-$ диэлектрическая проницаемость вакуума. Проводимость $\sigma_{1}$, обозначаемая в дальнейшем $\sigma$, зависит как от частоты, так и от температуры. Как и во всех изученных нами ранее $R \mathrm{Mn}_{2} \mathrm{O}_{5}[1-4]$, проводимость в ЕСМО обладает подобной частотной дисперсией (чем выше частота, тем выше проводимость). Согласно принятой картине дисперсии проводимости неоднородных сред, содержащих локальные области в однородной матрице [30], бездисперсионная низкочастотная проводимость характеризует сквозную проводимость матрицы $\sigma_{\mathrm{DC}}$. Зависящая же от частоты проводимость $\sigma_{\mathrm{AC}}$ (чем выше частота, тем выше проводимость) относится к локальной проводимости отдельных областей. Соотношение локальной и сквозной проводимостей, а также интервал температур существования областей с локальной проводимостью, удобно характеризовать относительной локальной проводимостью $\sigma_{\mathrm{loc}}=\left(\sigma_{\mathrm{AC}}-\sigma_{\mathrm{DC}}\right) / \sigma_{\mathrm{DC}}[30]$. Температурночастотные зависимости относительных локальных проводимостей для различных осей ЕСМО приведены на вставках к рис. $1, b, d$ и $f$.

Вдоль осей $a$ (рис. $1, b)$ и $c$ (рис. $1, f)$ наблюдаются качественно подобные зависимости для проводимостей ЕСМО. Из этих рисунков видно, что имеются два интервала температур, в которых изменяется характер частотной дисперсии проводимости. При низких температурах вплоть до некоторых температур, зависящих от ориентации осей кристалла, с ростом частоты проводимость уменьшается, т.е. при этих температурах $\sigma_{\mathrm{DC}}>\sigma_{\mathrm{AC}}$. При нарастании температуры величины $\sigma_{\mathrm{AC}}$ увеличиваются и при неких температурах, зависящих от частоты, пересекают низкочастотную проводимость $\sigma_{\mathrm{DC}}$. Чем выше частоты, тем при более высоких температурах наблюдаются такие пересечения. Температуры, при которых $\sigma_{\mathrm{AC}}$ сравниваются с $\sigma_{\mathrm{DC}}$, подчиняются закону Аррениуса $\omega=\omega_{0} \exp \left(-E_{A} / k T_{m}\right)$, (где $\omega-$ частота, а $T_{m}-$ температура, при которой $\sigma_{\mathrm{AC}}=\sigma_{\mathrm{DC}}, E_{A}-$ энергия активации на границах локальных областей). Это дает возможность определить активационные барьеры на границах этих низкотемпературных локальных областей для осей $a$ и $c$ кристалла (см. вставки к рис. $1, b$ и $f)$. Как видно, вдоль этих осей имеются добавочные высокотемпературные максимумы $\sigma_{\text {loc }}$, смещения температур которых в зависимости от частоты 

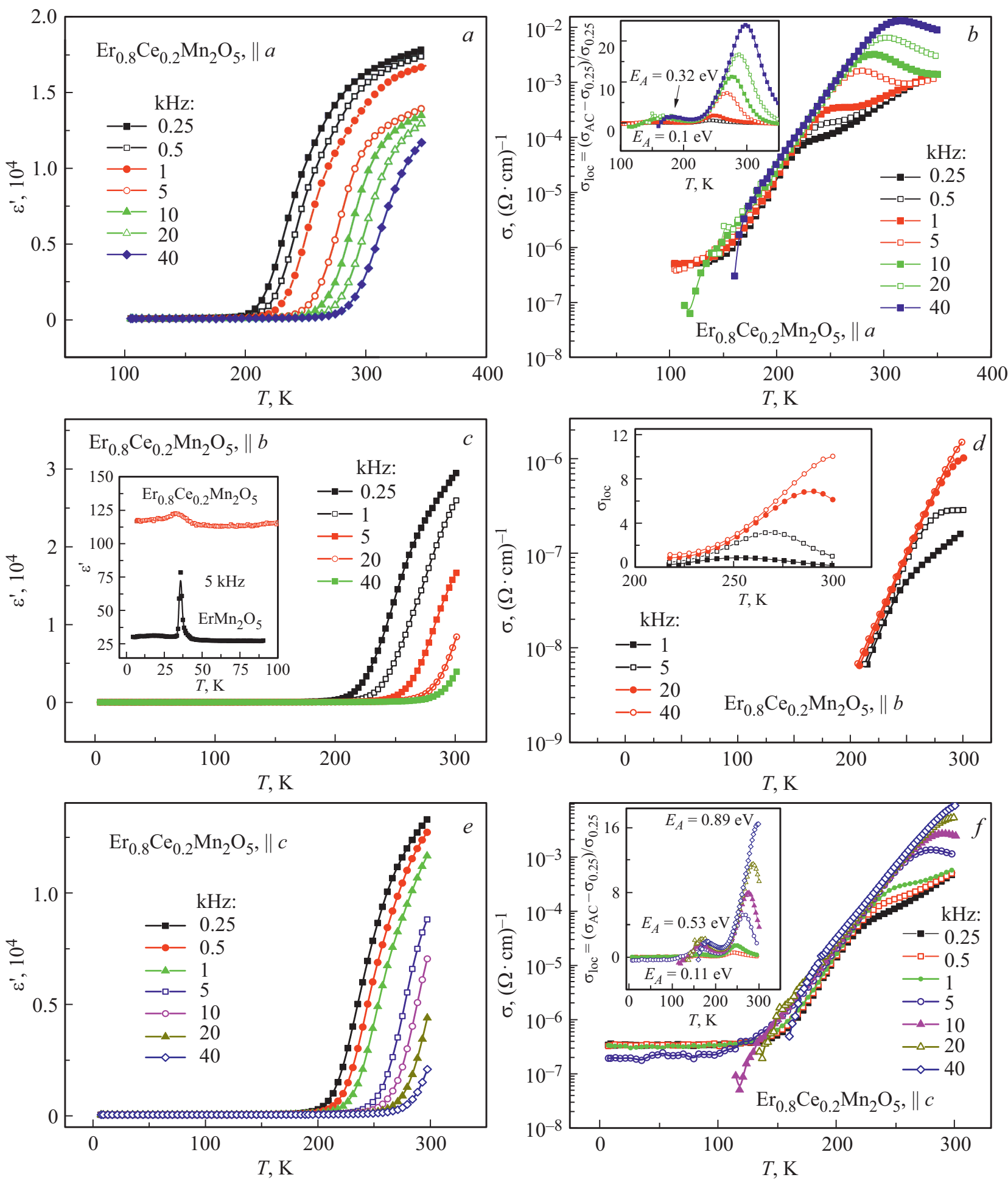

Рис. 1. Температурные зависимости диэлектрической проницаемости $\varepsilon^{\prime}$ для ряда частот вдоль осей $a, b, c$ (рис. $1, a, c, e$ соответственно) и проводимости $\sigma$ вдоль этих же осей (рис. $1, b, d$ и $f$ соответственно) для $\mathrm{Er}_{0.8} \mathrm{Ce}_{0.2} \mathrm{Mn}_{2} \mathrm{O}_{5}$. На вставке к рис. $1, c$ демонстрируются в большем масштабе, температурные зависимости $\varepsilon^{\prime}$ для $\operatorname{Er}_{0.8} \mathrm{Ce}_{0.2} \mathrm{Mn}_{2} \mathrm{O}_{5}$ и $\operatorname{ErMn}_{2} \mathrm{O}_{5}$ в интервале температур $5-80 \mathrm{~K}$ на частоте $5 \mathrm{kHz}$. На вставках к рис. $1, b, d$ и $f$ приведены температурные зависимости локальной проводимости. Частоты указаны на рисунках.

также подчиняются закону Аррениуса, что позволяет определить активационные барьеры на границах таких высокотемпературных локальных областей. Они также указаны на графиках для локальных проводимостей. Таким образом, наблюдение в ЕСМО локальной проводимости вдоль осей $a$ и $c$ с различными активаци- 

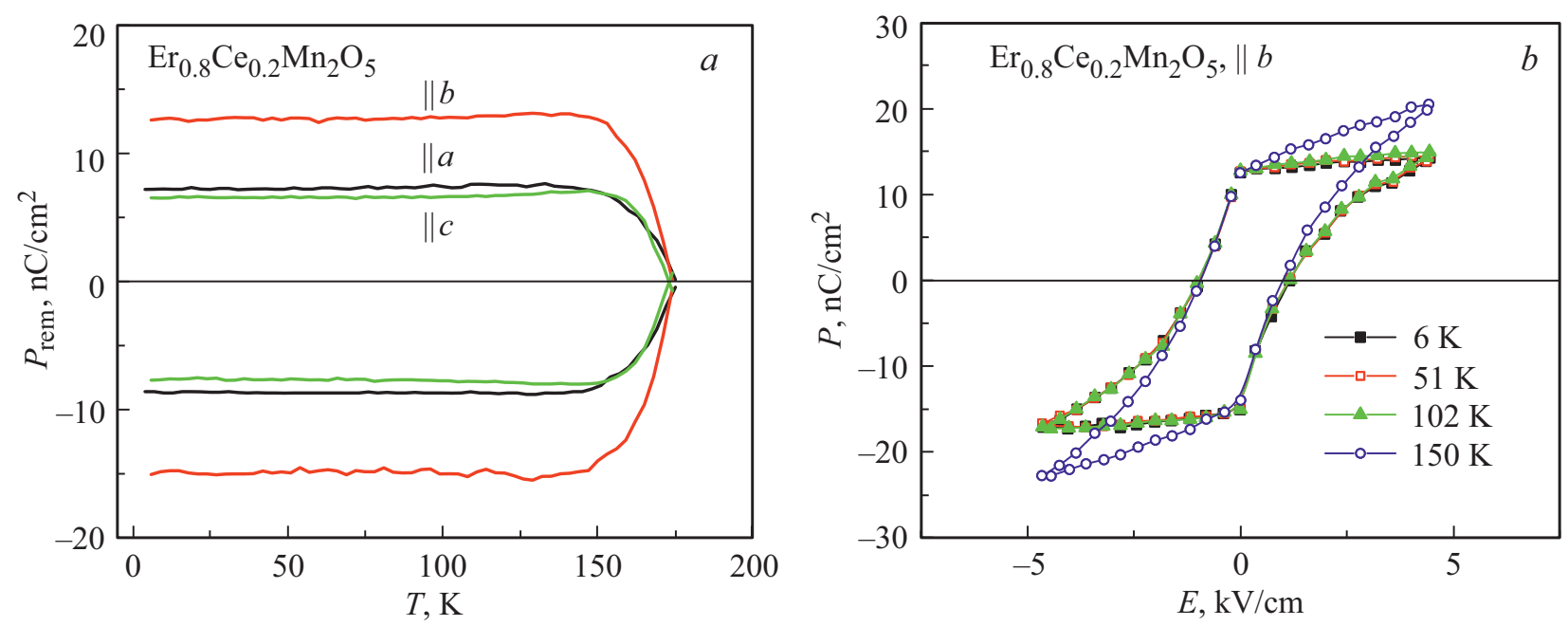

Рис. 2. Температурные зависимости остаточной поляризации $P_{\text {rem }}$ вдоль различных осей $\operatorname{Er}_{0.8} \mathrm{Ce}_{0.2} \mathrm{Mn}_{2} \mathrm{O}_{5}$ (рис. 2,a) и набор петель гистерезиса электрической поляризации при различных температурах вдоль оси $b$ (рис. $2, b$ ).

онными барьерами свидетельствует о наличии областей фазового расслоения двух типов. Мы относим области с отрицательной локальной проводимостью $\sigma_{\mathrm{loc}}$ (т.е. с низкочастотной сквозной проводимостью $\sigma_{\mathrm{DC}}$, превышающей высокочастотные $\sigma_{\mathrm{AC}}$ проводимости) к локальным областям фазового расслоения, формирующимся внутри марганцевой подсистемы, находящейся при низких температурах под высокими барьерами решетки, обусловленными ионами $\mathrm{Er}$ и $\mathrm{Ce}$. Как было показано в $[2,3,11,12,14-16]$ для $R \mathrm{Mn}_{2} \mathrm{O}_{5} \quad(R=\mathrm{Eu}, \mathrm{Gd}, \mathrm{Bi})$ при низких температурах области фазового расслоения занимают малый объем кристалла. С ростом температуры увеличивается вероятность для носителей заряда преодолевать более высокотемпературные барьеры в решетке, обусловленные ионами $\mathrm{Er}$ и Се. При этом формируются более высокотемпературные локальные области фазового расслоения (рис. $1, b$ и $f$ ). Отметим, что проводимость в этих областях также обусловлена переносами $e_{g}$ электронов между ионами марганца различной валентности, но уже в областях с более высокими барьерами. Таким образом локальные области фазового расслоения Мn подсистемы обладают различными свойствами при достаточно низких температурах и при более высоких температурах после преодоления решеточного барьера. Основными характеристиками этих областей являются температурные зависимости локальных проводимостей $\sigma_{\mathrm{loc}}$ (вставки на рис. $1, b$ и $f$ ) и электрической поляризации (рис. $2, a$ и $b$ ).

На рис. $2, a$ и $b$ приведены соответственно температурные зависимости остаточной поляризации петель электрической поляризации $P_{\text {rem }}$ вдоль всех осей ЕСМО и, для примера, петли электрической поляризации для ряда характерных температур вдоль оси $b$. Отметим, что форма петель гистерезиса вдоль всех осей подобна, а о величинах поляризации можно судить по температурным зависимостям остаточной поляризации (рис. 2,a).
Величины проводимостей вдоль осей $a$ и $c$ (рис. $1, b$ и $f$ ) близки, активационные барьеры на границах локальных областей при температурах ниже $200 \mathrm{~K}$ (определяемые смещениями с частотой первых максимумов относительной локальной проводимости) достаточно велики $(0.32 \mathrm{eV}$ вдоль оси а и $0.53 \mathrm{eV}$ вдоль оси $c)$. Величины $\sigma_{\mathrm{loc}}$ в максимумах вблизи $175 \mathrm{~K}$ составляют 1.8 вдоль оси $a$ и 2.3 вдоль оси $c$, что определяет близость величин остаточной поляризации: $7.3 \mathrm{nC} / \mathrm{cm}^{2}$ вдоль оси $a$ и $6.5 \mathrm{nC} / \mathrm{cm}^{2}$ вдоль оси $c$ (рис. 2,a). Высокие барьеры на границах низкотемпературных локальных областей вдоль осей $a$ и $c$ при повышенной проводимости на всех частотах (рис. $1, b$ и $f$ ) обеспечивают появление относительно высокой концентрации носителей под этими барьерами. Минимальная остаточная поляризация вдоль оси $c$ в ЕСМО (рис. 2, $a$ ) при более высоком барьере на границах указывает на то, что вероятнее всего под этими барьерами имеется концентрация электронов, превышающая количество пар ионов $\mathrm{Mn}^{3+}-\mathrm{Mn}^{4+}$, которые перезаряжаются электронами. Эти избыточные электроны частично экранируют внутренние электрические поля внутри локальных областей и понижают поляризацию.

Вдоль же оси $b$ в ЕСМО наблюдается аномально низкая проводимость и мы не можем измерить при $T<200 \mathrm{~K}$ ни $\sigma$, ни $\sigma_{\mathrm{loc}}$, также как не можем определить барьеры на границах локальных областей. Однако, о существовании таких областей на рис. $1, d$ свидетельствует характер частотной дисперсии, характерный для локальных областей (чем выше частота, тем выше проводимость) (рис. $1, d$ ) для проводимости при $T>200 \mathrm{~K}$ (в обычном суперпараэлектрическом состоянии). В замороженном же суперпараэлектрическом состоянии (при $T<175 \mathrm{~K}$ ) величина остаточной поляризации вдоль оси $b$ в ЕСМО максимальна $P_{\text {rem }} \approx 12,6 \mathrm{nC} / \mathrm{cm}^{2}$ (рис. $2, a$ ), в то время как в ЕМО вдоль этой оси она была минимальна 
$\left(P_{\mathrm{rem}} \approx 2 \mathrm{nC} / \mathrm{cm}^{2}\right)$, но существовала до более высокой температуры $270 \mathrm{~K}$ [1]. Мы полагаем, что в направлении оси $b$ в ЕСМО имеется значительно более сильное искажение решетки по сравнению с EMO [1]. Это необходимо отнести за счет влияния ионов Се, так как ионы $\mathrm{Er}$ максимально искажают решетку вдоль оси $c$ и в ЕМО максимальная поляризация $7.5 \mathrm{nC} / \mathrm{cm}^{2}$ наблюдается именно вдоль оси $c[1]$. При этом основной вклад в поляризацию вдоль оси $b$ ECMO вносят искажения решетки в локальных областях от ионов Се и внутреннее поле в этих областях и активационные барьеры на их границах должны быть максимальны. При этом, казалось бы, должно происходить накопление большой концентрации носителей заряда внутри локальных областей и соответственно экранировки поляризации. Но из-за аномально малой проводимости вдоль оси $b$ (рис. $1, d)$ экранировка внутренних полей в этих областях незначительна.

В работах [1-4] для $R \mathrm{Mn}_{2} \mathrm{O}_{5}(R=\mathrm{Gd}, \mathrm{Bi}, \mathrm{Er}, \mathrm{Tb})$ было показано, что замороженное суперпараэлектрическое состояние, для которого наблюдались петли электрической поляризации, существует до температуры, при которой выполняется условие $k T \approx E_{A}$ активационному барьеру на границах полярных областей. При более высоких температурах области фазового расслоения Mn-подсистемы продолжают существовать, находясь в обычном суперпараэлектрическом состоянии, для которого электрическая поляризация исчезает. Это согласуется с теоретической работой [17].

Рост диэлектрической проницаемости ЕСМО над стабильным низкотемпературным фоновом значением также начинается вблизи $175 \mathrm{~K}$. Обращает на себя внимание, что анизотропия наблюдаемых величин диэлектрической проницаемости $\varepsilon^{\prime}$ при $T>175 \mathrm{~K}$ не соответствует наблюдаемой анизотропии проводимостей. Этот факт также указывает на то, что величины $\varepsilon^{\prime}$ в большей степени определяются структурными искажениями в решетке, наиболее сильными вдоль оси $b$.

Таким образом, в ЕСМО вдоль осей $a$ и $c$ наблюдается четкая корреляция в диэлектрических свойствах и проводимости локальных полярных областей фазового расслоения с индуцированной этими областями электрической поляризации. Такая же корреляция наблюдалась в ранее изученных $R \mathrm{Mn}_{2} \mathrm{O}_{5}(R=\mathrm{Gd}, \mathrm{Bi}, \mathrm{Er}, \mathrm{Tb})$ вдоль всех осей кристаллов [1-4].

Качественно иная ситуация возникает в ЕСМО вдоль оси $b$. Как следует из вставки рис. $1, c$ разбавление ЕМО ионами Се сильно размывает низкотемпературный сегнетоэлектрический переход. Это свидетельствует о том, что нарушается зарядовое упорядочение чередующихся ферромагнитных и антиферромагнитных пар ионов $\mathrm{Mn}^{3+}-\mathrm{Mn}^{4+}$ вдоль этой оси. В ЕМО (как и в любом $R \mathrm{Mn}_{2} \mathrm{O}_{5}$ ) такое упорядочение приводило в обменнострикционному механизму низкотемпературного сегнетоэлектрического упорядочения. Именно большая вероятность переноса $e_{g}$-электронов между соседними парами ионов $\mathrm{Mn}^{3+}-\mathrm{Mn}^{4+}$ приводила к повышенному фону сквозной проводимости вдоль оси $b$ в ЕМО, что уменьшало локальную проводимость и остаточную поляризацию [1]. Согласно рис. $1, d$ проводимости ЕСМО вдоль оси $b$ на всех частотах, при температурах выше $200 \mathrm{~K}$, на два порядка меньше. Это свидетельствует либо о значительном разрушении вдоль оси $b$ зарядового упорядочения, либо о возникновении довольно сильного искажения кристаллического поля вдоль оси $b$ ионами Се. Наблюдение при низких температурах в ЕСМО остаточной поляризации постоянной величины вдоль оси $b$ вплоть до $125 \mathrm{~K}$ (рис. 2, $a$ ) свидетельствует о существовании подобных локальных полярных областей фазового расслоения от самых низких температур, но под очень высокими барьерами в решетке, формируемыми ионами Се. Таким образом, вдоль оси $b$ преобладающим фактором является искажение кристаллического поля вдоль этой оси ионами Се.

Как уже отмечалось выше, в наших кристаллах ионы Се обладают переменной валентностью $\mathrm{Ce}^{+3.75}$ (т.е. большая часть ионов Се проявляют себя как $\mathrm{Ce}^{4+}$ и частично как $\mathrm{Ce}^{3+}$ ). Рассмотрим теперь источники искажения кристаллического поля ионами Се. Напомним, что $R$-ионы в $R \mathrm{Mn}_{2} \mathrm{O}_{5}$ имеют нецентральное пятиугольное кислородное окружение, подобное ионам $\mathrm{Mn}^{3+}$. При этом они расположены в слоях $z=0 c$. В соседних плоскостях с $z=0.25 c$ и $z=0.75 c$, содержатся ионы $\mathrm{Mn}^{4+}$ с октаэдрическими кислородными окружениями. В результате замещения $\mathrm{Er}^{3+}$ ионом $\mathrm{Ce}^{4+}$ появляется избыточный электрон, который в соседних слоях перезаряжает ион $\mathrm{Mn}^{4+}+e=\mathrm{Mn}^{3+}$, что является причиной увеличения концентрации локальных полярных областей фазового расслоения в ЕСМО по сравнению с ЕМО. Заметим также, что несмотря на близость ионных радиусов ионов $\mathrm{Ce}^{4+}$ и $\mathrm{Er}^{3+}$ (в шестиугольной координации 0.87 и $0.89 \AA$ соответственно [24]) происходит добавочное искажение кристаллического поля в ЕСМО по сравнению с ЕМО по двум причинам. Во-первых, из-за увеличения концентрации полярных областей, а во-вторых из-за того, что часть ионов $\mathrm{Er}^{3+}$, хотя и с меньшей вероятностью, замещаются более крупными ионами $\mathrm{Ce}^{3+}$ (с ионным радиусом $1.01 \AA$ [24]. Эти ионы содержат уединенные пары $6 s^{2}$-электронов на внешней оболочке. Как известно, ионы, содержащие уединенные пары $6 s^{2}$-электронов на наружных оболочках сильно локально искажают решетку [22]. Таким образом, в твердом растворе ЕСМО по сравнению с исходным EMO концентрация локальных полярных областей фазового расслоения больше, и они находятся в другом искаженном кристаллическом поле, что изменяет диэлектрические свойства и электрическую поляризацию в ЕСМО по сравнению с ЕМО. Поляризации этих двух кристаллов различаются по величинам, анизотропии и температурам существования. Как оказалось, ионы $\mathrm{Er}^{3+}$ искажают кристаллическое поле вдоль оси $c$, а ионы $\mathrm{Ce}^{+3.75}$ вдоль оси $a$ и $b$. За формирование локальных областей фазового расслоения ответственна подсистема ионов $\mathrm{Mn}$, расположенная в кристаллических полях, 
формируемых $R$-ионами. Источником же полярности самих марганцевых подсистем являются следующие два фактора. Внутри областей фазового расслоения двойной обмен, связанный с переносом $e_{g}$-электронов между парами ионов $\mathrm{Mn}^{3+}-\mathrm{Mn}^{4+}$, приводит к тому, что в позициях ионов $\mathrm{Mn}^{4+}$ (кислородных октаэдрах) оказываются ян-теллеровские ионы $\mathrm{Mn}^{3+}$, которые деформируют эти октаэдры. В свою очередь, меньшие по размеру ионы $\mathrm{Mn}^{4+}$ (по сравнению с ионами $\mathrm{Mn}^{3+}$ ) оказываются в нецентральных пятиугольных пирамидах и также добавочно их искажают [1-4].

\section{2. Диэлектрические свойства и электрическая поляризация B $\mathrm{Tb}_{0.8} \mathrm{Ce}_{0.2} \mathrm{Mn}_{2} \mathrm{O}_{5}$}

На рис. $3, a-f$ для $\mathrm{Tb}_{0.8} \mathrm{Ce}_{0.2} \mathrm{Mn}_{2} \mathrm{O}_{5}$ (ТСМО) приведены температурные зависимости диэлектрической проницаемости $\varepsilon^{\prime}$ для ряда частот вдоль осей $a, b, c$ (рис. 3, $a, c, e$ соответственно) и проводимости $\sigma$ вдоль этих же осей (рис. $3, b, d$ и $f$ ). На вставке рис. $3, c$ для ТСМО приведены величины $\varepsilon^{\prime}$ вдоль оси $b$ при низких температурах в большем масштабе для ряда частот. Как видно, разбавление ионов Тb ионами Се приводит к практически полному разрушению низкотемпературного сегнетоэлектрического упорядочения вдоль оси $b$ (ср. с рис. 4,c [1]). Значительные нарастания проницаемостей $\varepsilon^{\prime}$ на различных частотах вдоль всех осей также начинаются с $T \sim 175 \mathrm{~K}$, как это было и в ЕСМО. Вблизи комнатной температуры максимальное значение $\varepsilon^{\prime}$ наблюдается вдоль оси $c\left(4 \cdot 10^{4}\right)$, минимальное вдоль оси $b\left(6 \cdot 10^{3}\right)$.

Как видно из рис. $3, b, d$ и $f$ проводимости и их анизотропия в ТСМО отличаются от этих величин в $\mathrm{TbMn}_{2} \mathrm{O}_{5}$ (ТМO) [1]. Качественно температурно-частотные зависимости проводимости ТСМО вдоль всех осей подобны. Но величины проводимости существенно различаются: вдоль осей $a$ и $b$ они близки, в то время как вдоль оси $c$ проводимость на два порядка меньше.

В ТСМО также наблюдаются два типа локальных областей: низкотемпературных в интервале температур $(5-175 \mathrm{~K})$ и высокотемпературных при $T>175 \mathrm{~K}$. В интервале температур $175-225 \mathrm{~K}$ вдоль всех осей наблюдаются экспоненциально нарастающие (линейные в логарифмическом масштабе) проводимости. На вставках рис. $3, b, d$ и $f$ представлены температурные зависимости локальной проводимости $\sigma_{\text {loc. }}$ При температурах ниже $125 \mathrm{~K}$ локальная проводимость отрицательная (т.е. сквозная низкочастотная проводимость матрицы больше высокочастотных проводимостей областей фазового расслоения). Начиная с самых низких температур все более высокочастотные проводимости при более высоких температурах начинают пересекать независящую от температуры сквозную проводимость, превышая ее. При этом формируются максимумы локальной проводимости вблизи $150 \mathrm{~K}$, высокотемпературные склоны которых при приближении к $T \sim 200 \mathrm{~K}$ приближаются к сквозной проводимости (т.е. $\sigma_{\text {loc }}$ уменьшается, приближаясь к нулю вблизи $200 \mathrm{~K}$ ). Как отмечалось выше, условием превращения замороженного суперпараэлектрического состояния в обычное суперпараэлектрическое, и исчезновения остаточной поляризации является $\sigma_{\mathrm{loc}} \approx 0$, когда $k T \approx E_{A}$ на границах локальных областей.

На рис. 4, $a$ приведены температурные зависимости остаточной поляризации вдоль различных осей ТСМО. На рис. 4, $b$ представлены петли электрической поляризации ТСМО для ряда характерных температур.

Как видно из сравнения рис. 4, $a$ со вставками к рис. $3, b, d$ и $f$ температуры исчезновения остаточной поляризации вдоль различных осей в ТСМО оказались несколько меньше, чем температуры, при которых $\sigma_{\text {loc }}$ стремится к нулю. Они близки к $150 \mathrm{~K}$. При этом максимальная температура наблюдается вдоль оси $a$, минимальная вдоль оси $c$. Рассмотрим причину этого более подробно.

Проводимость вдоль оси $a$ максимальна, барьер на границах локальных областей, ответственных за возникновение остаточной поляризации, $0.3 \mathrm{eV}$ (рис. $3, b$ и вставка к нему). Близкая проводимость имеется вдоль оси $b$, но барьер на границах областей $0.2 \mathrm{eV}$ (рис. $3, d$ ). Это обеспечивает подобные фоновые значения низкотемпературной остаточной поляризации вдоль осей $a$ и $b$, но уменьшает температуру ее существования вдоль оси $b$ (рис. $4, a)$. Это согласуется с уменьшением активационного барьера $E_{A}$ вдоль оси $b$ по сравнению с осью $a$.

Вдоль оси $c$ проводимость на два порядка меньше по сравнению с осями $a$ и $b$ (рис. $3, f$ ), что уменьшает концентрацию областей фазового расслоения, понижая при этом величину остаточной поляризации, индуцируемую этими областями. Однако, оба активационных барьера на границах локальных областей вдоль оси $c$, обозначенные на вставке рис. $3, f$, выше чем вдоль оси $b$ : от пересечений $\sigma_{\mathrm{AC}}$ с $\sigma_{\mathrm{DC}}$ в 4 раза, от смешений первого максимума вблизи $150 \mathrm{~K}$ в 1.5 раза. Казалось бы, это должно увеличить температуру существования поляризации вдоль оси с по сравнению с осью $b$. На самом деле, она уменьшается, хотя и незначительно (рис. $4, a)$.

Причина этого заключается в том, что увеличение низкотемпературного барьера вдоль оси $c$ в четыре раза приводит к более резкому нарастанию проводимости локальных областей под высокотемпературным барьером $(0.3 \mathrm{eV})$. Действительно, локальная проводимость в максимумах (с активационным барьером $0.3 \mathrm{eV}$ ) вдоль оси с превышает 2, в то время как вдоль осей $a$ и $b$ она меньше (см. вставки к рис. $3, b, d, f)$. В результате избыточные электроны под барьером $0.3 \mathrm{eV}$ должны его частично экранировать, понижая реальную температуру существования остаточной поляризации вдоль оси $c$ (рис. $4, a)$.

Соотношение обеих активационных барьеров на границах низкотемпературных локальных областей в ЕСМО и ТСМО сильно отличаются. Эти барьеры в ЕСМО значительно больше, чем в ТСМО. В результате, 

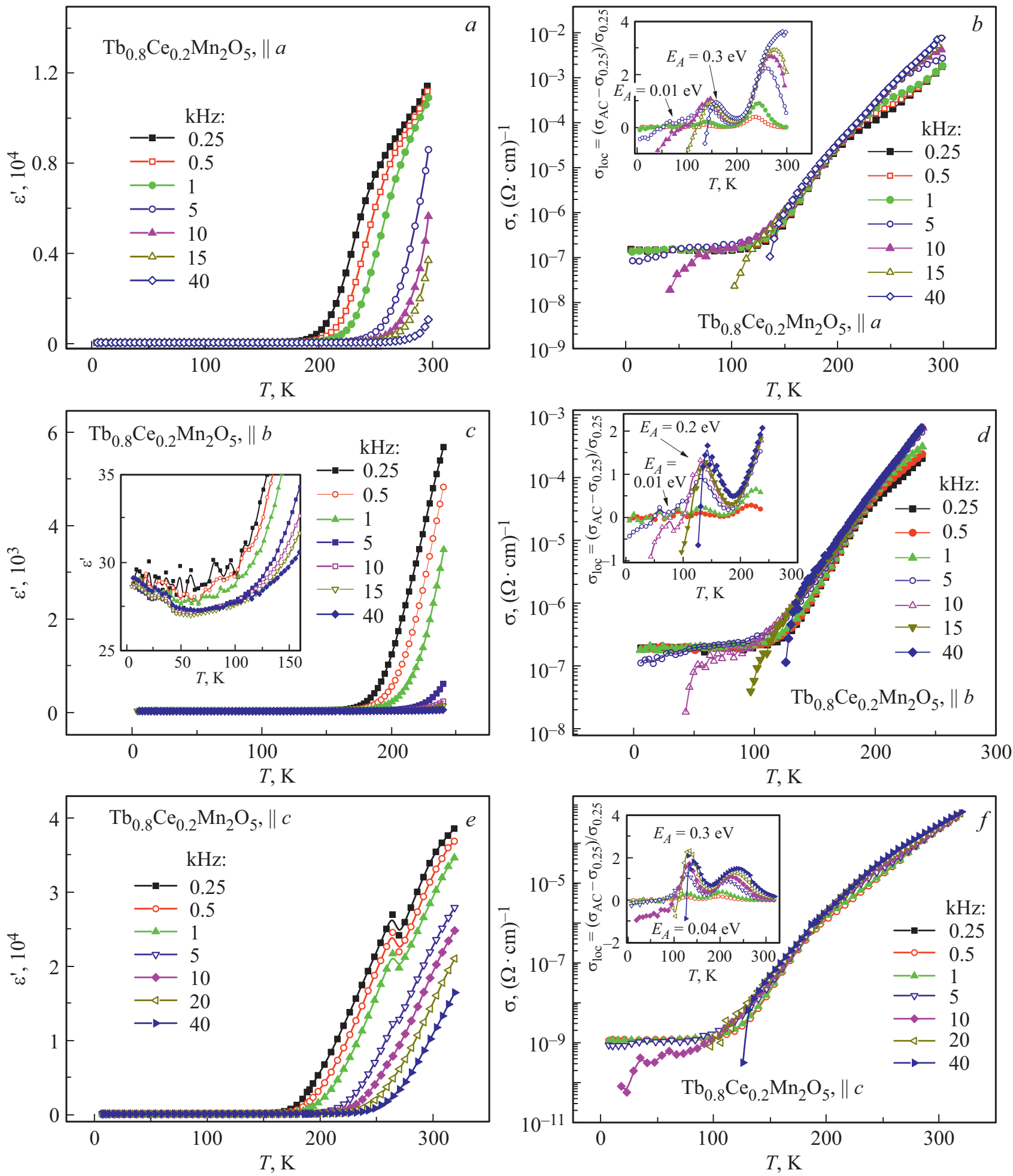

Рис. 3. Температурные зависимости диэлектрической проницаемости $\varepsilon^{\prime}$ для ряда частот вдоль осей $a, b, c$ (рис. $3, a, c, e$ соответственно) и проводимости $\sigma$ вдоль этих же осей (рис. $3, b, d$ и $f$ соответственно) для $\mathrm{Tb}_{0.8} \mathrm{Ce}_{0.2} \mathrm{Mn}_{2} \mathrm{O}_{5}$. На вставках к рис. $3, b, d$ и $f$ приведены температурные зависимости локальной проводимости. Частоты указаны на рисунках.

в ТСМО увеличивается концентрация носителей экранирующих и так не высокие барьеры, что уменьшает как величины остаточных поляризаций вдоль всех осей в ТСМО, так и понижает температуры их существования.
В ЕСМО ионы $\mathrm{Er}$ сильно искажают решетку вдоль оси $c$, увеличивая барьеры на границах областей вдоль этой оси. Это при повышенной проводимости вдоль оси $c$ экранирует барьеры, понижая поляризацию. В то 

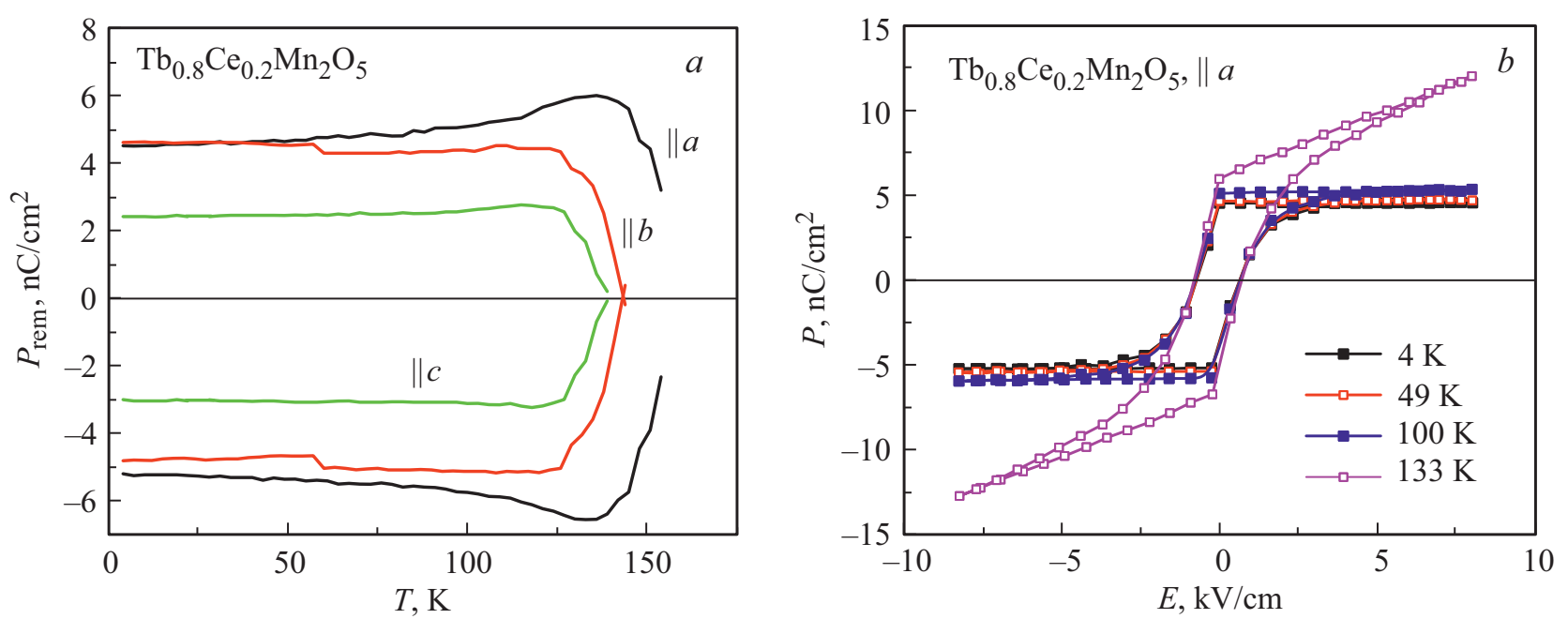

Рис. 4. Температурные зависимости остаточной поляризации $P_{\text {rem }}$ вдоль различных осей $\mathrm{Tb}_{0.8} \mathrm{Ce}_{0.2} \mathrm{Mn}_{2} \mathrm{O}_{5}($ рис. $3, a)$ и набор петель гистерезиса электрической поляризации при различных температурах вдоль оси $a$ (рис. $3, b$ ).

же время ионы Се в ЕСМО сильно искажают решетку вдоль оси $b$, нарушая зарядовое упорядочение вдоль этой оси, резко уменьшая проводимость. В результате в ЕСМО максимальная поляризация наблюдается вдоль оси $b$.

В ТСМО ионы Тb искажают решетку в плоскости $a b$ при близких проводимостях вдоль осей $a$ и $b$, а ионы Се сильно искажают решетку вдоль оси $c$, на два порядка уменьшая проводимость областей фазового расслоения вдоль этой оси, что уменьшает концентрацию областей фазового расслоения и поляризацию. В результате, величины поляризаций в ТСМО оказались значительно меньше, чем в ЕСМО. При этом максимальная поляризация наблюдается вдоль оси $a$.

\section{4. Заключение}

Как следует из работы [1] и настоящего исследования, полярные области фазового расслоения в $R \mathrm{Mn}_{2} \mathrm{O}_{5}$ и $R_{0.8} \mathrm{Ce}_{0.2} \mathrm{Mn}_{2} \mathrm{O}_{5}(R=\mathrm{Er}, \mathrm{Tb})$ формируются в подсистеме ионов $\mathrm{Mn}$, состоящей из ионов $\mathrm{Mn}^{3+}$ и $\mathrm{Mn}^{4+}$, определенным образом распределенных в кристалле. Основными процессами при формировании полярных областей фазового расслоения является перенос электронов между ионами $\mathrm{Mn}^{3+}$ и $\mathrm{Mn}^{4+}$, на которые существенно влияют величины кристаллических полей, формируемых $R$ - и Се-ионами. Эти локальные полярные области в центросимметричной матрице кристаллов формируют суперпараэлектрическое состояние, которое ниже некоторых температур имеет свойства замороженного суперпараэлектрического состояния, отклик которого на приложенное внешнее электрическое поле имеет вид петель гистерезиса электрической поляризации с остаточной поляризацией. Было установлено, что величины поляризации, а также температуры их существования определяются характером кристаллических полей, создаваемых R ионами. При этом разбавление ионов $\mathrm{Er}$ и Ть ионами Се сильнее изменяет анизотропию кристаллических полей в ЕСМО и ТСМО по сравнению с ионами $\mathrm{Er}$ и $\mathrm{Tb}$ в EMO и ТМО соответственно, величины и температуры существования электрической поляризации.

\section{Благодарности}

Авторы благодарны за частичную финансовую поддержку работы Российскому фонду фундаментальных исследований (гранты 18-32-00241, 20-02-00667) и Программе 1.4 Президиума РАН „Актуальные проблемы физики низких температур“.

\section{Конфликт интересов}

Авторы заявляют, что у них нет конфликта интересов.

\section{Список литературы}

[1] Б.Х. Ханнанов, Е.И. Головенчиц, В.А. Санина. ФТТ 62, 257 (2020).

[2] B.Kh. Khannanov, V.A. Sanina, E.I. Golovenchits, M.P. Scheglov. Письма в ЖЭТФ 103, 274 (2016).

[3] B.Kh. Khannanov, V.A. Sanina, E.I. Golovenchits, M.P. Scheglov. JMMM 421, 326 (2017).

[4] В.А. Санина, Б.Х. Ханнанов, Е.И. Головенчиц, М.П. Щеглов. ФТТ 60, 531 (2018).

[5] N. Hur, S. Park, P.A. Sharma, J.S. Ahn, S. Guba, S-W. Cheong. Nature (London) 429, 392 (2004).

[6] Y. Noda, H. Kimura, M. Fukunaga, S. Kobayashi, I. Kagomiya, K. Kohn. J. Phys.: Condens. Matter 20, 434206 (2008).

[7] P.G. Radaelli, L.C. Chapon. J. Phys.: Condens. Matter 20, 434213 (2008).

[8] P.G. de Gennes. Phys. Rev. 118, 141 (1960).

[9] Л.П. Горьков. УФН 168, 655 (1998).

[10] J. van den Brink, D.I. Khomskii. J. Phys.: Condens. Matter 20, 434217 (2008). 
[11] V.A. Sanina, E.I. Golovenchits, V.G. Zalesskii, S.G. Lushnikov, M.P. Scheglov, S.N. Gvasaliya, A. Savvinov, R.S. Katiyar, H. Kawaji, T. Atake. Phys. Rev. B 80, 224401 (2009).

[12] V.A. Sanina, E.I. Golovenchits, B.Kh. Khannanov, M.P. Scheglov, V.G. Zalesskii. Письма в ЖЭТФ 100, 451 (2014).

[13] М.Ю. Каган, К.И. Кугель. УФН 171, 533 (2001).

[14] В.А. Санина, Е.И. Головенчиц, В.Г. Залесский. Письма в ЖЭТФ 95, 429 (2012).

[15] V.A. Sanina, E.I. Golovenchits, V.G. Zalesskii. J. Phys.: Condens. Matter 24, 346002 (2012).

[16] В.А. Санина, Б.Х. Ханнанов, Е.И. Головенчиц. ФТТ 59, 1932 (2017).

[17] M.D. Glinchuk, E.A. Eliseev, A.N. Morozovska. Phys. Rev. B 78, 134107 (2008).

[18] V. Baledent, S. Chattopadhyay, P. Fertey, M.B. Lepetit, M. Greenblatt, B. Wanklyn, F.O. Saouma, J.I. Jang, P. FouryLeylekian. Phys. Rev. Lett. 114, 117601 (2015).

[19] N. Lee, C. Vecchini, Y.J. Choi, L.C. Chapon, A. Bombardi, P.G. Radaelli, S.W. Cheong,.Phys. Rev. Lett. 110, 137203 (2013).

[20] B.Kh. Khannanov, V.A. Sanina, E.I. Golovenchits. J. Phys. Conf. Ser. 572, 012046 (2014).

[21] К.П. Белов, А.К. Звездин, А.М. Кадомцева, Р.З. Левитин. Ориентационные переходы в редкоземельных магнетиках. Наука, М. (1979).

[22] N.A. Hill, K.M. Rabe. Phys. Rev. B 59, 8759 (1999).

[23] Z.H. Sun, B.L. Cheng, S. Dai, K.J. Jin, Y.L. Zhou, Y.B. Lu, Z.H. Chen, G.Z. Yang. J. Appl. Phys. 99, 084105 (2006).

[24] R.D. Shannon. Acta Crystallogr. A 32, 751 (1976).

[25] В.А. Санина, Л.М. Сапожникова, Е.И. Головенчиц, Н.В. Морозов. ФТТ 30, 3015 (1988).

[26] А.В. Бабинский, Е.И. Головенчиц, Н.В. Морозов, Л.М. Сапожникова. ФТТ 34, 108 (1992).

[27] J.F. Scott, L. Kammerdiner, L.M. Parris, S. Traynor, V. Ottenbacher, A. Shawabkeh, W.F. Oliver. J. Appl. Phys. 64, 787 (1988).

[28] M. Fukunaga, Y. Noda. J. Phys. Soc. J 77, 0647068 (2008).

[29] S.M. Feng, Y.S. Chai, J.L. Zhu, N. Manivannan, Y.S. Oh, L.J. Wang, Y.S. Yang, C.Q. Jin, Kee Hoon Kim. New J. Phys. 12, 073006 (2010).

[30] A.R. Long. Adv. Phys. 31, 587 (1982).

Редактор Т.Н. Василевская 